\title{
Managing Heterogeneity in a Grid Parallel Haskell
}

\author{
A. Al Zain ${ }^{1}$, P.W. Trinder ${ }^{1}$, H-W. Loidl ${ }^{2}$, and G.J. Michaelson ${ }^{1}$ \\ 1 School of Mathematical and Computer Sciences, Heriot-Watt University, Riccarton, \\ Edinburgh EH14 4AS, UK \\ \{ceeatia, trinder, greg\}@macs.hw.ac.uk \\ 2 Ludwig-Maximilians-Universität München, Institut für Informatik, D 80538 \\ München, Germany \\ hwloidl@inf ormatik. uni-muenchen.de
}

\begin{abstract}
Grid-GUM is a distributed virtual shared-memory implementation of a high-level parallel language for computational Grids. While the implementation delivers good speedups on multiple homogeneous clusters with low-latency interconnect, on heterogeneous clusters, however, poor load balance limits performance. Here we present new load management mechanisms that combine static and partial dynamic information to adapt to heterogeneous Grids. The mechanisms are evaluated by measuring four non-trivial programs with different parallel properties, and show runtime improvements between $17 \%$ and $57 \%$, with the most dynamic program giving the greatest improvement.
\end{abstract}

\section{Introduction}

Hardware price/performance ratios and improved middleware and network technologies make cluster computing and computational Grids increasingly attractive. These architectures are typically heterogeneous in the sense that they combine processing elements with different CPU speeds and memory characteristics. Parallel programming on such heterogeneous architectures is more challenging than on classical homogeneous high performance architectures.

Rather than requiring the programmer to explicitly manage low level issues such as heterogeneity we advocate a high-level parallel programming language, specifically $\mathrm{GpH}$, where the programmer controls only a few key parallel coordination aspects. The remaining coordination aspects including heterogeneity are dynamically managed by a sophisticated runtime environment, GUM. GUM has been engineered to deliver good performance on classical HPCs and clusters [6]. Grid-GUM is a port of GUM to computational Grids using the Globus Toolkit, the de-facto standard. As Grid-GUM implements a virtual shared-memory over a wide-area network, it is perhaps surprising that it gives good performance in some instances, e.g. on homogeneous low-latency multiclusters [1]. However for heterogeneous architectures load management emerges as the performancelimiting issue. 
This paper presents Grid-GUM2, incorporating new load management mechanisms for heterogeneous architectures. The new mechanisms are decentralised, obtain complete static information during start up, and then cheaply propagate partial dynamic information during execution. The existing load management techniques in Grid-GUM are described in Section 2. The design of the new thread, load and communication management mechanisms is given in Section 3. The effectiveness of the new mechanisms for heterogeneous clusters is investigated using four non-trivial programs from a range of application areas, and with varying degrees of irregular parallelism and using both data parallel and divideand-conquer paradigms in Section 4. Related work is discussed in Section 5, and we conclude in Section 6 .

\section{$2 \quad \mathrm{GpH}$ and Grid-GUM}

GpH (Glasgow parallel Haskell) [7] is a modest and conservative extension of Haskell 98 with very high level coordination, i.e. control of parallel execution. Parallel and sequential composition primitives introduce and synchronise threads. Evaluation strategies are polymorphic higher-order functions that abstract over the primitives to provide parameterisable, reusable high level coordination control.

Grid-GUM Grid-GUM extends the existing GUM memory management, and thread management techniques. In particular, it implements a virtual shared heap over a wide-area network 8 . The communication management in Grid-GUM is similar to GUM, but uses MPICH-G2, a Grid-enabled implementation of the MPI standard [5], and hence the Globus Toolkit as middle-ware.

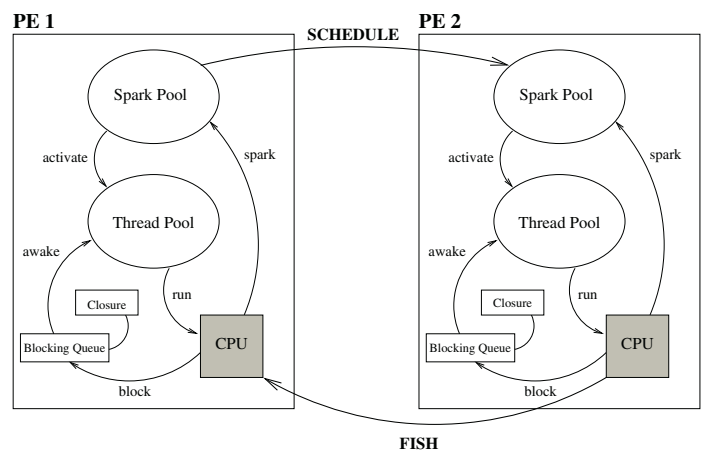

Fig. 1. Interaction of the components of a GUM processing element

Grid-GUM uses an evaluate-and-die thread management model with sparks, i.e. pointers to graph structures, to represent potential parallelism. Sparks are generated by an explicit par construct in the program and maintained by the runtime-system in a flat spark pool. A sparked expression may be executed by an independent thread. However, if a thread needs the value of the expression, and 
no other thread is evaluating it, the demanding thread will perform the computation itself. This behaviour is called thread subsumption because the potentially parallel work is inlined by another thread.

Figure 1 illustrates the load management mechanism in Grid-GUM, depicting the logical components on each Processing Element (PE) of the Grid-GUM abstract machine. When activated a spark causes a new thread to be generated. Threads that are not currently being executed reside in the thread pool. When the CPU is idle, and the thread pool is empty, a spark will be activated to generate a thread. If a running thread blocks on unavailable data, it is added to the blocking queue of that node until the data becomes available.

The thick arrows between the PEs in Figure 1 show load management messages exchanged in Grid-GUM. Initially all processors, except for the main PE, will be idle, with no local sparks available. PE2 sends a FISH message to a random-chosen PE. On arrival of this message, PE1 will search for a spark and, if available, send it to PE2. This mechanism is usually called work stealing or passive load distribution, since an idle processor has to ask for work. Grid-GUM also improves load distribution by using Limited Thread mechanism which includes specifying a hard limit on the total number of live threads, i.e. runnable or blocked threads in the thread pool. To summarise, the Grid-GUM load mechanism deals with both locating work (Figure 2, a), and handling work requests (Figure 2,b), where these activities are performed in the main scheduler loop between thread time slices.

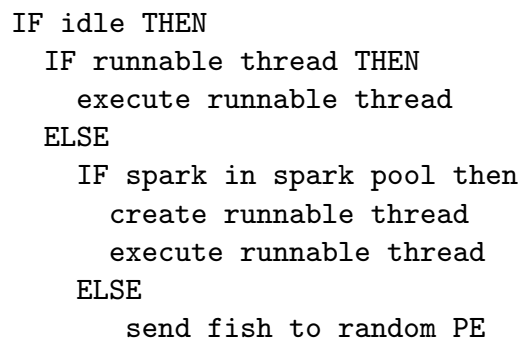

Fig[2]. Work locating

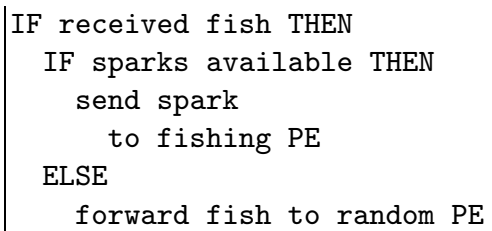

Fig,2b. Work request handling

Fig. 2. Grid-GUM Load Management

Grid-GUM performance In earlier work we have investigated the performance of GpH programs on Grid-GUM, with varying numbers of clusters and interconnection latencies [1]. We find that Grid-GUM delivers good and predictable speedups for several configurations, e.g. multiple homogeneous clusters with a low-latency interconnect, or programs that perform little communication on multiple clusters with a high latency interconnect. However other configurations, including heterogeneous clusters, Grid-GUM gives poor performance due to poor load management. 


\section{Grid-GUM2}

Distributed load management assigns processes (or threads) to PEs in a multiPEs system and can be classified as depicted in Figure 3. In static load management, PEs are assigned tasks at compile time, i.e. before the execution begins. In contrast, dynamic load management assumes that limited knowledge about the processes and PEs is available a priori, and load management decisions are made during execution. Dynamic scheduling is subdivided into two categories: a) centralised where a single $\mathrm{PE}$ is responsible for scheduling, and b) decentralised where all PEs participate in the scheduling. Distributed techniques can either operate in a passive mode where idle PEs request work, or in an active mode where PEs are assigned work without a prior request. This section presents

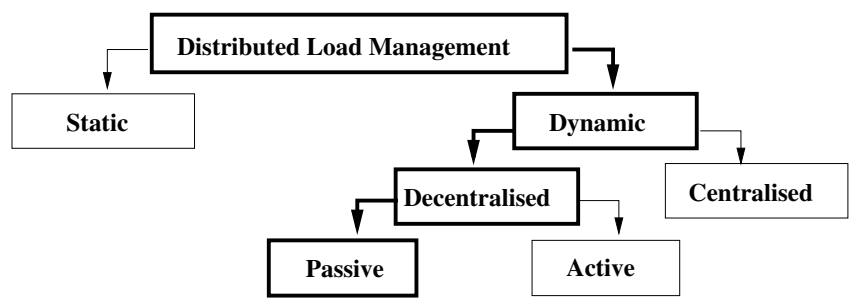

Fig. 3. Classifying Grid-GUM2 Load Management

Grid-GUM2, a new adapted load scheduling to improve Grid-GUM performance. Grid-GUM2 load management is dynamic, decentralised and mostly passive using complete static and partial dynamic information about PEs, processes and network. The salient features of Grid-GUM2 are as follows.

Targeted Load Management: The challenge in load managment is to efficiently and effectively distribute the available work using dynamic information about PE CPU speed and loads, with minimal overheads. In Grid-GUM, if a PE is idle, it sends a FISH message to a randomly-chosen PE. However, given the increased heterogeneity of computational Grids, Grid-GUM2 sources work from a PE that has a high load relative to its speed.

Load information must be maintained dynamically, but it is prohibitively expensive to regularly broadcast complete load information to every $\mathrm{PE}$ in a large computational Grid. Grid-GUM2 uses a lightweight approach maintaining partial dynamic PE information at almost no cost. Each PE maintains a table PEDynamic that contains partial timestamped dynamic information about the PEs, currently just their loads as depicted in Figure 5 The PEDynamic table is included with every message sent between PEs, and the receiving PE updates its table with more recent information. The additional cost of sending the table is minimal for the high bandwidth interconnects in typical computational Grid.

Specialised Thread Management: Grid-GUM has a parameter that specifies the minimum number of sparks that should be available in each $\mathrm{PE}$, the low- 
watermark. If the number of sparks in a $\mathrm{PE}$ falls below the low-watermark it issues a FISH message to obtain more work. Such a uniform strategy for all PEs must be adapted for heterogeneous clusters. Our design is to maintain a local low-watermark for each PE, with higher watermarks for fast PEs. This encourages fast PEs to be more aggressive about obtaining work than slower PEs. However, local low watermarks are not get implemented in the system measured in the next section.

Variable Latency Communication Management: The interconnects between PEs in a computational Grid have varying static and dynamic latencies, e.g. the latency between PEs in the same cluster is much less than that between PEs on different clusters, and both are affected by network traffic. Our

\begin{tabular}{|c|c|c|}
\hline PE & CPU Speed & Time Stamp \\
\hline A & $550 \mathrm{MHz}$ & $13: 40: 01$ \\
\hline D & $550 \mathrm{MHz}$ & $13: 45: 00$ \\
\hline C & $350 \mathrm{MHz}$ & $12: 40: 03$ \\
\hline B & $350 \mathrm{MHz}$ & $13: 44: 03$ \\
\hline F & $350 \mathrm{MHz}$ & $14: 40: 03$ \\
\hline
\end{tabular}

Fig. 4. PEStatic Table

\begin{tabular}{|c|c|c|}
\hline PE & Load & time_stamp \\
\hline A & 2000 & $14: 13: 49$ \\
\hline D & 3000 & $14: 13: 59$ \\
\hline B & 10000 & $14: 12: 22$ \\
$\bullet$ & $\bullet$ & $\bullet$ \\
$\bullet$ & $\bullet$ & $\bullet$ \\
\hline
\end{tabular}

Fig. 5. PEDynamic Table

\begin{tabular}{|c|c|c|}
\hline PE & Latency & Last Update \\
\hline F & $0.75 \mathrm{msec}$ & $12: 45: 20$ \\
\hline G & $2.05 \mathrm{msec}$ & $12: 24: 50$ \\
\hline C & $10.00 \mathrm{msec}$ & $12: 50: 25$ \\
$:$ & $:$ & $:$ \\
\hline
\end{tabular}

Fig. 6. Communic. Table

mechanism prefers to obtain work and hence data from PEs that currently have low communication latency. Grid-GUM2 maintains communication information at almost no cost. Each PE has a table Communication that contains partial timestamped dynamic information about the communication, currently just the latency to each PE as depicted in Figure 6. The latency of every message is estimated, timestamped and recorded in the Communications table with the startup messages initialising the table. The problems of maintaining synchronised distributed clocks is minimised by measuring message send and receive times as time elapsed since the program start.

Grid-GUM2 introduces a novel mechanism to deal with cases where there is no work available on local, i.e low-latency, PEs. When an idle PE requests work from a PE residing outside its cluster, then a scheduling decision is automatically taken on the basis of minimising intra-cluster communication. One can distinguish two scenarios: a) if the request originated from relatively powerful cluster, then multiple sparks, i.e. several work items, are returned in a SuperSchedule message; b) if the request originated from a relatively weak cluster then the request is served as usual, i.e. as a single spark. Note that the idea of Super-Schedule is not evaluated in this work since it is under implementation.

The core of Grid-GUM2 load management can be summarised as work location (Figure 3,a), and work request handling (Figure 3,b). 


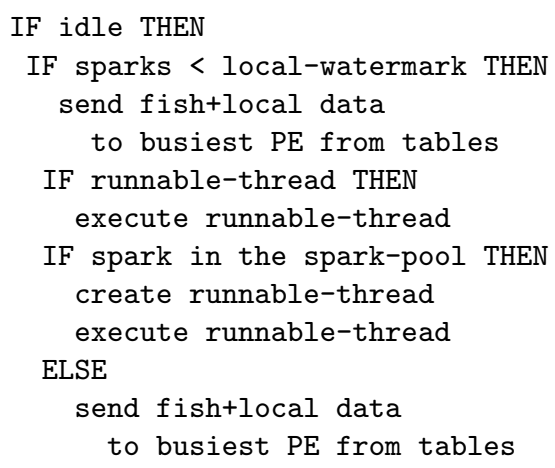

Fig 3 ,a. Work locating

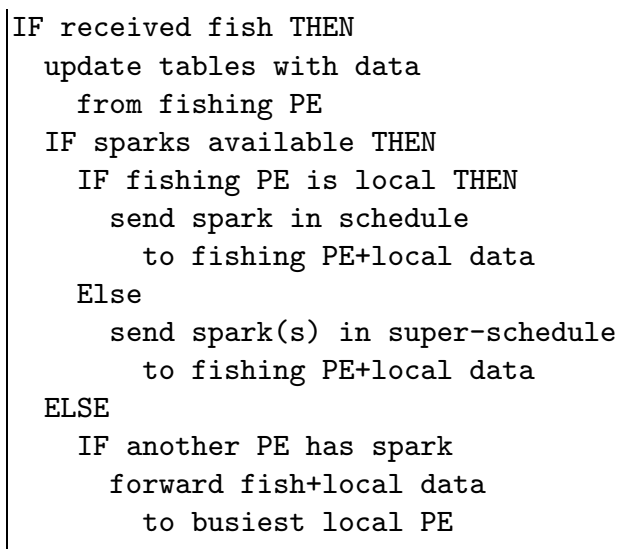

Fig, 3. b. Work request handling

Fig. 7. Grid-GUM2 Load Management

\section{Performance Comparison}

Table 1 compares the runtime for four $\mathrm{GpH}$ programs with different paradigms, and parallelism regularities, on multiple heterogeneous clusters with moderate latency interconnect. The four programs are measured in this experiment are: queens which places chess pieces on a board; sumEuler which computes the sum of the Euler totient values of a list; linSolv which finds an exact solution of a linear system of equations; raytracer which calculates a 2D image of a given scene of $3 \mathrm{D}$ objects by tracing all rays in a given grid, or window. All run-times are the median of three executions to ameliorate the impact of the operating system and shared network interaction. The experiments were performed on eight machines: four machines with fast CPU speed $(1395 \mathrm{MHz})$ and four with slow CPU speed (534 MHz).

Overall, the dynamic adaptive scheduling of Grid-GUM2 consistently shows the best performance on heterogeneous Grid multi-clusters.

Due to limited space we focus on investigating the behaviour of one benchmark program, raytracer. In particular, raytracer has highly irregular execution, and consequently is very sensitive to changes in parallel environment.

Table 1. Programs characteristics and performance

\begin{tabular}{|c|c|c|c|c|c|c|}
\hline \multirow[t]{2}{*}{ Program } & \multirow{2}{*}{$\begin{array}{l}\text { Application } \\
\text { Area }\end{array}$} & \multirow[t]{2}{*}{ Paradigm } & \multirow[t]{2}{*}{ Regularity } & \multicolumn{3}{|c|}{ Runtimes \& Improvement \% } \\
\hline & & & & $\begin{array}{l}\text { Grid- } \\
\text { Basic }\end{array}$ & $\begin{array}{l}\text { Grid-GUM + } \\
\text { Thread Limi }\end{array}$ & Grid-GUM2 \\
\hline queens & AI & Div-Conq. & Regular & 668 & $33350 \%$ & $31053 \%$ \\
\hline sumEuler & Nume. Analysi & Data Para. & Limit irreg. & 570 & $34339 \%$ & $27951 \%$ \\
\hline linSolv & Symb. algebra & Data Para. & Limit irreg. & 217 & $306-40 \%$ & $18017 \%$ \\
\hline raytracer & Graphic & Data Para. & High irreg. & 1340 & $81439 \%$ & $57257 \%$ \\
\hline
\end{tabular}




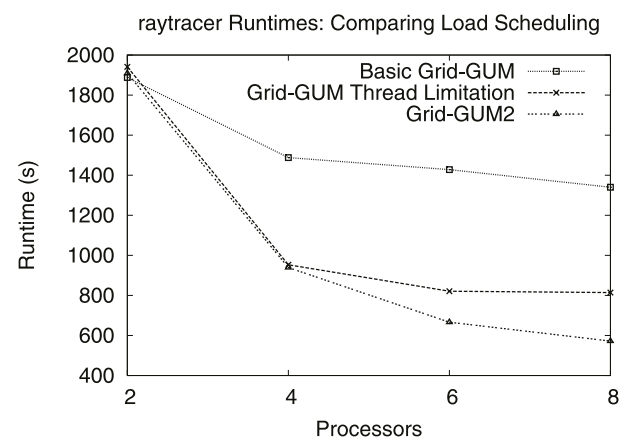

Fig. 8. Runtimes for raytracer

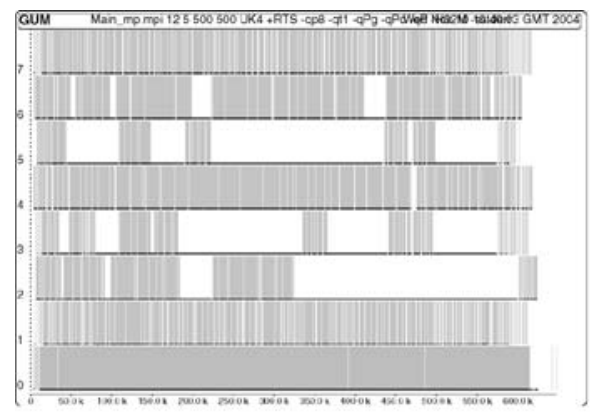

a) Grid-GUM

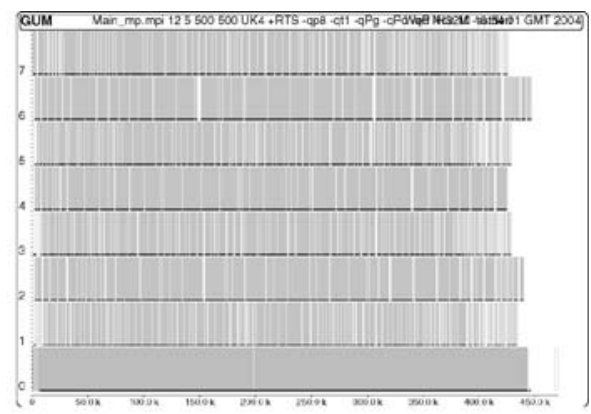

b) Grid-GUM2

Fig. 9. per-PE activity profile for raytracer

Figure $\$$ compares the runtime achieved for the raytracer using different load management mechanisms on a heterogeneous environment, where there are the same numbers of slow and fast machines. For more than 4 PEs the best results are achieved for Grid-GUM2 with better scalability and decreasing runtime than basic Grid-GUM and Grid-GUM with thread limitation.

Figure 9 shows per-PE activity profile for raytracer, with execution on four fast machines $(0,2,4,6)$, and four slow machines $(1,3,5,7)$. A per-PE activity profile shows the behaviour for each of the PEs (y-axis) over execution time (x-axis). Each PE is visualised as a horizontal line, with darker shades of gray indicating a larger number of runnable threads. Gaps in the horizontal lines indicate idleness. Figure 9 a depicts the performance on Grid-GUM using a limit of 1 for the number of live threads. This small limit is chosen because it is the only one that has any impact on a heterogeneous architecture. Figure 9 b depicts the performance on Grid-GUM2. All PEs in Figure 9b are uniformly loaded, and finish at the same time, in contrast to the PEs in Figure 9] a have numerous idle periods. Figure 9 a also shows long idle periods at the middle of the computation, where only a small amount of parallelism is available. Here, blocking on data 
that is evaluated in the PEs with slow CPU speed will cause the PEs with fast CPU speed to remain idle until new work is obtained. Note that the runtime drops from $814 \mathrm{~s}$ to $572 \mathrm{~s}$, which almost (30\%) improvement.

\section{$5 \quad$ Related Work}

The most closely related work is the ConCert system [4, which translates a subset of ML to machine code, for execution on a Grid architecture. In contrast to our work, parallelism is expressed via explicit synchronisation. Alt et al apply skeletons to computational Grids [3]. This work focuses on providing the application user with skeletons to capture common patterns of Grid abstractions. However, our aim is to provide more general programming language support for parallelism through an implementation that incorporates new implicit dynamic coordination-management strategies. Aldinucci et al also apply skeletons to computational Grids 2. This work focuses on providing a skeleton to centralise load management in the Grid environment. However, our aim is to solve load scheduling on the Grid by developing a dynamic decentralised load schedule.

\section{Conclusion}

The Grid-GUM2 extension to the Grid-GUM runtime environment has been produced to efficiently and automatically manages data and work on a multi-cluster Grid environment. Achieving good performance was made possible by using a high-level parallel language and completely managing distribution at the runtime environment level . As future work we plan to measure the behaviour of Grid-GUM2 using varying number of clusters and interconnect latencies. We are also considering building an analytic model of Grid-GUM2.

\section{References}

1. A. Al Zain, P. Trinder, H-W. Loidl, and G. Michaelson. Grid-GUM: Towards GridEnabled Haskell. In IFL'04 - Intl. Workshop on the Implementation of Functional Languages, Draft Proceedings, Lübeck, Germany, September 2004.

2. M. Aldinucci, M. Dnelutto, and Dünnweber. Optimization Techniques for Implementing Parallel Sckeletons in Grid Environments. In CMPP'04 - Intl. Workshop on Constructive Methods for Parallel Programming, Stirling, Scotland, July 2004.

3. M. Alt, H Bischof, and S. Gorlatch. Program Development for Computational Grids Using Skeletons and Performance Prediction. In CMPP'02 - Int. Workshop on Constructive Methods for Parallel Programming. Dagstuhl, Berlin, June 2002.

4. B-Y. Evan Chang, K. Crary, M. DeLap, R. Harper, J. Liszka, T. Murphy VII, and F. Pfenning. Trustless Grid Computing in ConCert. In In Proceedings of the GRID 2002 Workshop, volume 2536 of LNCS. Springer-Verlag, 2001.

5. N. Karonis, B. Toonen, and I. Foster. MPICH-G2: A grid-enabled implementation of the message passing interface. J. of Parallel and Distributed Computing, 2003. 
6. H-W. Loidl, F. Rubio, N. Scaife, K. Hammond, S. Horiguchi, U. Klusik, R. Loogen, G. J. Michaelson, R. Pe na, Á. J. Rebón Portillo, S. Priebe, and P. W. Trinder. Comparing Parallel Functional Languages: Programming and Performance. Higherorder and Symbolic Computation, 16(3), 2003.

7. P.W. Trinder, K. Hammond, H-W. Loidl, and S.L. Peyton Jones. Algorithm + Strategy = Parallelism. J. of Functional Programming, 8(1):23-60, January 1998.

8. P.W. Trinder, K. Hammond, J.S. Mattson Jr., A.S. Partridge, and S.L. Peyton Jones. GUM: a Portable Parallel Implementation of Haskell. In PLDI'96 - Conf. on Programming Language Design and Implementation, Philadephia, USA, 1996. 